\title{
Compensation Decoding of Space Time Frequency Block Codes
}

\author{
Min Zhang, A.D.S. Jayalath, Thushara D. Abhayapala, David Smith, and Chandra Athaudage
}

\begin{abstract}
A novel compensation decoding scheme for a given space time frequency linear block code is presented, exploiting the simplicity of zero forcing equalization, and special characteristics of the precoding matrix. The proposed decoding procedure is relatively simple and straightforward in comparison to maximum likelihood decoding (MLD) and sphere decoding (SD). The bit-error-rate performance of the proposed scheme is better than zero forcing decoding and close to MLD and SD for low to medium signal-to-noise ratio range.
\end{abstract}

Index Terms-STF coding, MIMO-OFDM, STF decoding.

\section{INTRODUCTION}

W IRELESS communication system with multiple input multiple output (MIMO) and orthogonal frequency division multiplexing (OFDM) is an attractive candidate for next generation wireless networks. In order to achieve full space-time-frequency (STF) diversity of MIMO-OFDM systems, subcarrier grouping or frequency grouping [1]-[3] is usually used to reduce the system complexity and reuse welldeveloped space-time (ST) coding schemes. Moreover, STF coding schemes proposed in [1]-[5] are capable of achieving full STF diversities using maximum-likelihood decoding (MLD) or sphere decoding (SD). The complexity of SD and MLD increases dramatically with employing more subcarriers. On the other hand, zero forcing decoding (ZFD) [6] has much simpler decoding procedure at the expense of performance compared with MLD and SD, alternatively requires redundant subcarriers and a complex matrix inversion. Hence there is a trade-off between system performance and complexity, which we propose to address by a new method simplifying the decoding procedure into element by element decoding for a slightly modified STF coding scheme presented in [1].

\section{Channel Model And Coding}

Here we consider a frequency selective MIMO-OFDM system with $N_{t}$ transmitters, $N_{r}$ receivers, $F$ subcarriers and $K$ symbol intervals of time where $N_{t}=K=2$. The channel is assumed to be quasi-static over $K$ symbol intervals. Each pair of transmitter and receiver has $L+1$ resolvable delay paths. By subcarrier grouping we assume that $P$ uniformly

Manuscript received February 22, 2007. The associate editor coordinating the review of this letter and approving it for publication was Murat Uysal. This work is supported by Australia Research Council Discovery Grant DP0558865.

M. Zhang, A.D.S. Jayalath, and T.D. Abhayapala are with The Australian National University and National ICT Australia Limited (email: min.zhang@rsise.anu.edu.au; Dhammika.Jayalath@nicta.com.au; Thushara.Abhayapala@anu.edu.au).

D. Smith is with National ICT Australia Limited (email: David.Smith@nicta.com.au).

C. Athaudage is with the University of Melbourne (email: crna@unimelb.edu.au).

Digital Object Identifier 10.1109/LCOMM.2007.070276. separated subcarriers are employed for the precoding scheme in each group.

It has been proved that the full space frequency diversities of $N_{t} P$ and rate one can be achieved only if $P \leq(L+1)$ and $N_{t}=2$ for the precoding scheme in [1]. In this paper we adopt a part of the precoding procedure in [1] to develop a simpler, element by element decoding method for the case of two transmitters. Applying Alamouti coding for ST domains, the MIMO-OFDM channel equation at the $p$ th subcarrier is given by

$$
\left\{\begin{array}{l}
\mathbf{Y}_{1 p}=\sqrt{\frac{\rho}{2}}\left(s_{1 p} \mathbf{H}_{1 p}+s_{2 p} \mathbf{H}_{2 p}\right)+\mathbf{n}_{1 p} \\
\mathbf{Y}_{2 p}=\sqrt{\frac{\rho}{2}}\left(-s_{2 p}^{*} \mathbf{H}_{1 p}+s_{1 p}^{*} \mathbf{H}_{2 p}\right)+\mathbf{n}_{2 p}
\end{array} ;\right.
$$

where $\mathbf{Y}_{k p}$ for $k \in[1,2]$ and $p \in[1, \cdots, P]$ is $1 \times N_{r}$ received signal vector by $N_{r}$ receivers during the $k$ th symbol interval at the $p$ th subcarrier where $*$ is the complex conjugation operation. Complex scalars $s_{1 p}$ and $s_{2 p}$ are symbols transmitted through the $p$ th subcarrier, where $p \in[1, \cdots, P]$. $\mathbf{H}_{t p}$ where $t \in[1,2]$ and $p \in[1, \cdots, P]$ is $1 \times N_{r}$ channel frequency response vector of wireless propagation from the $t$ th transmitter to $N_{r}$ receivers at the $p$ th subcarrier. We assume that there is perfect channel state information (CSI) of $\mathbf{H}_{t p}$ at the receiver, but no CSI at the transmitter. $\rho$ is the average signal-to-noise ratio (SNR) at each receiver with same transmission power, independent of the number of transmitters, receivers and subcarriers. $\mathbf{n}_{k p}$ is $1 \times N_{r}$ noise vector during the $k$ th symbol interval at the $p$ th subcarrier. Each entry of $\mathbf{n}_{k p}$ is the independent complex additive white Gaussian noise (AWGN) with zero mean and unit variance.

The optimal coding gain and full diversity can be achieved with a Vandermonde matrix [1], [2], [7]. However in this paper we propose to employ a real matrix $\boldsymbol{\Phi}$ in [8] with full frequency diversity and potentially suboptimal coding gain. The real matrix $\boldsymbol{\Phi}$ will simplify the decoding procedure specified in the next section. Hence we have

$$
\left[s_{11}, \cdots, s_{1 P}\right]=\mathbf{C}_{1} \boldsymbol{\Phi}, \quad\left[s_{21}, \cdots, s_{2 P}\right]=\mathbf{C}_{2} \boldsymbol{\Phi}
$$

where $\boldsymbol{\Phi}$ is $P \times P$ real matrix and $\boldsymbol{\Phi}_{p}$ is defined as the $p$ th column vector of $\boldsymbol{\Phi}$. Each entry of the codeword vectors $\mathbf{C}_{1}$ and $\mathbf{C}_{2}$ is a complex scalar chosen from a given constellation $\mathcal{A}$. They are defined as $\mathbf{C}_{1}=\left[c_{1}, \cdots, c_{P}\right]$ and $\mathbf{C}_{2}=$ $\left[c_{P+1}, \cdots, c_{2 P}\right]$ so that symbol transmission rate equals to unity for this STF block coding scheme. The real matrix $\boldsymbol{\Phi}$ is restricted by: $\boldsymbol{\Phi} \boldsymbol{\Phi}^{T}=\mathbf{I}$ and $\boldsymbol{\Phi}^{T} \boldsymbol{\Phi}=\mathbf{I}$ where ${ }^{T}$ is the transpose operation of matrix and $\mathbf{I}$ is defined as an identity matrix. From (2), transmitted symbol $s_{k p}$ for $k \in[1,2]$ and $p \in[1, \cdots, P]$ can be expressed as $s_{k p}=\mathbf{C}_{k} \boldsymbol{\Phi}_{p}$ which is substituted into (1). After applying a conjugate operation and combining equations for different subcarriers, we have an 
equivalent channel equation to (1) given by the following

$$
\tilde{\mathbf{Y}}=\left[\mathbf{C}_{1}, \mathbf{C}_{2}\right] \tilde{\mathbf{H}}+\tilde{\mathbf{N}}
$$

where

$$
\begin{aligned}
\tilde{\mathbf{Y}} & =\left[\begin{array}{lllll}
\mathbf{Y}_{11} & \mathbf{Y}_{21}^{*} & \ldots & \mathbf{Y}_{1 P} & \mathbf{Y}_{2 P}^{*}
\end{array}\right] \\
\tilde{\mathbf{H}} & =\left[\begin{array}{lllll}
\boldsymbol{\Phi}_{1} \mathbf{H}_{11} & \boldsymbol{\Phi}_{1} \mathbf{H}_{21}^{*} & \ldots & \boldsymbol{\Phi}_{P} \mathbf{H}_{1 P} & \boldsymbol{\Phi}_{P} \mathbf{H}_{2 P}^{*} \\
\boldsymbol{\Phi}_{1} \mathbf{H}_{21} & -\boldsymbol{\Phi}_{1} \mathbf{H}_{11}^{*} & \ldots & \boldsymbol{\Phi}_{P} \mathbf{H}_{2 P} & -\boldsymbol{\Phi}_{P} \mathbf{H}_{1 P}^{*}
\end{array}\right] \\
\tilde{\mathbf{N}} & =\left[\begin{array}{lllll}
\mathbf{n}_{11} & \mathbf{n}_{21}^{*} & \cdots & \mathbf{n}_{1 P} & \mathbf{n}_{2 P}^{*}
\end{array}\right]
\end{aligned}
$$

and $\tilde{\mathbf{N}}$ is complex AWGN.

\section{MIMO-OFDM DECODING}

Starting from (3), we propose a decoding scheme, compensation decoding (CD), for this particular STF block coding scheme. Multiplying both sides of (3) by $\tilde{\mathbf{H}}^{\dagger}$ and using the properties of $\boldsymbol{\Phi}$, we have

$$
\begin{aligned}
\tilde{\mathbf{Y}} \tilde{\mathbf{H}}^{\dagger}= & \tilde{\mathbf{N}} \tilde{\mathbf{H}}^{\dagger}+\left[\mathbf{C}_{1}, \mathbf{C}_{2}\right] \\
\times & {\left[\begin{array}{l}
\sum_{p=1}^{P}\left(\mathbf{H}_{1 p} \mathbf{H}_{1 p}^{\dagger}+\mathbf{H}_{2 p} \mathbf{H}_{2 p}^{\dagger}\right) \boldsymbol{\Phi}_{p} \boldsymbol{\Phi}_{p}^{T}, \mathbf{0}_{P \times P} \\
\mathbf{0}_{P \times P}, \sum_{p=1}^{P}\left(\mathbf{H}_{1 p} \mathbf{H}_{1 p}^{\dagger}+\mathbf{H}_{2 p} \mathbf{H}_{2 p}^{\dagger}\right) \boldsymbol{\Phi}_{p} \boldsymbol{\Phi}_{p}^{T}
\end{array}\right] }
\end{aligned}
$$

where ${ }^{\dagger}$ is complex transpose conjugate and $\mathbf{0}_{P \times P}$ is the $P \times P$ dimensional zero matrix. $\left(\mathbf{H}_{1 p} \mathbf{H}_{1 p}^{\dagger}+\mathbf{H}_{2 p} \mathbf{H}_{2 p}^{\dagger}\right)$ for any $p \in[1, \cdots, P]$ is a scalar and defined as $\tilde{h}_{p}$. We also define the matrix $\sum_{p=1}^{P} \tilde{h}_{p} \boldsymbol{\Phi}_{p} \boldsymbol{\Phi}_{p}^{T}$ in (4) as $\hat{\mathbf{H}}$. Hence $\hat{\mathbf{H}}=$ $\boldsymbol{\Phi} \mathcal{D}\left[\tilde{h}_{1}, \cdots, \tilde{h}_{P}\right] \boldsymbol{\Phi}^{T}$ where $\mathcal{D}$ constitutes a diagonal matrix with entries $\left[\tilde{h}_{1}, \cdots, \tilde{h}_{P}\right]$. Using the properties of $\boldsymbol{\Phi}$, we have

$$
\left\{\begin{array}{l}
\hat{\mathbf{H}}^{1 / 2}=\boldsymbol{\Phi} \mathcal{D}\left[\tilde{h}_{1}^{1 / 2}, \cdots, \tilde{h}_{P}^{1 / 2}\right] \boldsymbol{\Phi}^{T} \\
\hat{\mathbf{H}}^{-1 / 2}=\boldsymbol{\Phi} \mathcal{D}\left[\tilde{h}_{1}^{-1 / 2}, \cdots, \tilde{h}_{P}^{-1 / 2}\right] \boldsymbol{\Phi}^{T}
\end{array} ;\right.
$$

Note that $\tilde{\mathbf{N}} \tilde{\mathbf{H}}^{\dagger}$ in (4) is colored Gaussian noise, hence multiplying $\left(\tilde{\mathbf{H}} \tilde{\mathbf{H}}^{\dagger}\right)^{-1 / 2}$ by both sides of (4), we have

$$
\tilde{\mathbf{Y}} \tilde{\mathbf{H}}^{\dagger}\left[\begin{array}{cc}
\hat{\mathbf{H}}^{-1 / 2} & \mathbf{0}_{P \times P} \\
\mathbf{0}_{P \times P} & \hat{\mathbf{H}}^{-1 / 2}
\end{array}\right]=\left[\mathbf{C}_{1}, \mathbf{C}_{2}\right]\left[\begin{array}{cc}
\hat{\mathbf{H}}^{1 / 2} & \mathbf{0}_{P \times P} \\
\mathbf{0}_{P \times P} & \hat{\mathbf{H}}^{1 / 2}
\end{array}\right]+\tilde{\mathbf{N}}
$$

Because $\tilde{\mathbf{H}}^{\dagger}\left(\tilde{\mathbf{H}} \tilde{\mathbf{H}}^{\dagger}\right)^{-1 / 2}$ is a unitary matrix, $\tilde{\mathbf{N}}$ in (6) is still complex AWGN.

Both $\tilde{\mathbf{Y}} \tilde{\mathbf{H}}^{\dagger}$ and $\tilde{\mathbf{N}}$ are then split up into two vectors equally, so $\tilde{\mathbf{Y}} \tilde{\mathbf{H}}^{\dagger}=\left[\hat{\mathbf{Y}}_{1}, \hat{\mathbf{Y}}_{2}\right]$ and $\tilde{\mathbf{N}}=\left[\mathbf{N}_{1}, \mathbf{N}_{2}\right]$. Hence (6) can be rewritten as

$$
\left\{\begin{array}{c}
\hat{\mathbf{Y}}_{1} \hat{\mathbf{H}}^{-1 / 2}=\mathbf{C}_{1} \hat{\mathbf{H}}^{1 / 2}+\mathbf{N}_{1} \\
\hat{\mathbf{Y}}_{2} \hat{\mathbf{H}}^{-1 / 2}=\mathbf{C}_{2} \hat{\mathbf{H}}^{1 / 2}+\mathbf{N}_{2}
\end{array}\right.
$$

where $\mathbf{N}_{1}$ and $\mathbf{N}_{2}$ are complex AWGN.

Note that both $\hat{\mathbf{H}}^{-1 / 2}$ and $\hat{\mathbf{H}}^{1 / 2}$ are real matrices. Therefore above equation can be split up further into real and imaginary parts easily:

$$
\left\{\begin{array}{l}
\operatorname{Re}\left(\hat{\mathbf{Y}}_{1}\right) \hat{\mathbf{H}}^{-1 / 2}=\operatorname{Re}\left(\mathbf{C}_{1}\right) \hat{\mathbf{H}}^{1 / 2}+\operatorname{Re}\left(\mathbf{N}_{1}\right) \\
\operatorname{Im}\left(\hat{\mathbf{Y}}_{1}\right) \hat{\mathbf{H}}^{-1 / 2}=\operatorname{Im}\left(\mathbf{C}_{1}\right) \hat{\mathbf{H}}^{1 / 2}+\operatorname{Im}\left(\mathbf{N}_{1}\right) \\
\operatorname{Re}\left(\hat{\mathbf{Y}}_{2}\right) \hat{\mathbf{H}}^{-1 / 2}=\operatorname{Re}\left(\mathbf{C}_{2}\right) \hat{\mathbf{H}}^{1 / 2}+\operatorname{Re}\left(\mathbf{N}_{2}\right) \\
\operatorname{Im}\left(\hat{\mathbf{Y}}_{2}\right) \hat{\mathbf{H}}^{-1 / 2}=\operatorname{Im}\left(\mathbf{C}_{2}\right) \hat{\mathbf{H}}^{1 / 2}+\operatorname{Im}\left(\mathbf{N}_{2}\right)
\end{array}\right.
$$

In (8) we have split up (3) into four independent subequations. Hence we only use the first equation of (8) as an example and decode the real component of $\mathbf{C}_{1}$. The decoding of rest of sub-equations will follow the exact same procedure.
For simplicity, we denote that $\mathbf{Y}=\operatorname{Re}\left(\hat{\mathbf{Y}}_{1}\right), \mathbf{C}=\operatorname{Re}\left(\mathbf{C}_{1}\right)$ and $\mathbf{N}=\operatorname{Re}\left(\mathbf{N}_{1}\right)$ which is independent real AWGN with zero mean and 0.5 variance. Thus the first equation of (8) is represented by

$$
\mathbf{Y} \hat{\mathbf{H}}^{-1 / 2}=\mathbf{C} \hat{\mathbf{H}}^{1 / 2}+\mathbf{N}
$$

Multiplying $\hat{\mathbf{H}}^{-1 / 2}$ by both sides of (9), we have

$$
\mathbf{Y} \boldsymbol{\Phi} \mathcal{D}\left[\tilde{h}_{1}^{-1} \ldots \tilde{h}_{P}^{-1}\right] \boldsymbol{\Phi}^{T}=\mathbf{C}+\mathbf{N} \hat{H}^{-1 / 2}
$$

Then we are able to use the left side of (10) to get the first estimation $\tilde{\mathbf{C}}$ of $\mathbf{C}$ by hard decision on real domain of the constellation $\mathcal{A}$.

$\tilde{\mathbf{C}}$ might not be a good estimation because $\mathbf{N} \hat{\mathbf{H}}^{-1 / 2}$ in (10) is colored Gaussian noise and the decoding of $\tilde{\mathbf{C}}$ is simply based on ZFD. Hence if there exists a better estimation, then the estimation can be expressed as $(\tilde{\mathbf{C}}+\Delta \mathbf{C}) . \Delta \mathbf{C}$ is denoted as the compensation vector respective to $\tilde{\mathbf{C}}$. Then our new target is to search the non-zero compensation vector $\Delta \mathbf{C}$ so that we could get a better estimation $(\tilde{\mathbf{C}}+\Delta \mathbf{C}) . \Delta \mathbf{C}$ needs to satisfy

$$
\left\|\mathbf{Y} \hat{\mathbf{H}}^{-1 / 2}-(\tilde{\mathbf{C}}+\Delta \mathbf{C}) \hat{\mathbf{H}}^{1 / 2}\right\|^{2}<\left\|\mathbf{Y} \hat{\mathbf{H}}^{-1 / 2}-\tilde{\mathbf{C}} \hat{\mathbf{H}}^{1 / 2}\right\|^{2}
$$

which satisfies the condition of MLD of (9). If $\tilde{\mathbf{C}}$ is the decoding result from MLD, there is clearly no non-zero $\Delta \mathrm{C}$ satisfying (11). $\tilde{\mathbf{C}}+\Delta \mathbf{C}$ should be limited by the values of constellation $\mathcal{A}$.

We can simplify (11) as

$$
\begin{aligned}
& \Delta \mathbf{C} \boldsymbol{\Phi} \mathcal{D}\left[\tilde{h}_{1}, \cdots, \tilde{h}_{P}\right] \boldsymbol{\Phi}^{T} \Delta \mathbf{C}^{T}< \\
& 2 \Delta \mathbf{C}\left(\mathbf{Y}^{T}-\boldsymbol{\Phi} \mathcal{D}\left[\tilde{h}_{1}, \cdots, \tilde{h}_{P}\right] \boldsymbol{\Phi}^{T} \tilde{\mathbf{C}}^{T}\right)
\end{aligned}
$$

Define $P \times 1$ vector $\boldsymbol{\Lambda}$ as

$$
\begin{aligned}
\boldsymbol{\Lambda}=2 & \left(\mathbf{Y}^{T}-\mathbf{\Phi} \mathcal{D}\left[\tilde{h}_{1}, \cdots, \tilde{h}_{P}\right] \boldsymbol{\Phi}^{T} \tilde{\mathbf{C}}^{T}\right) \\
& \cdot /\left(\boldsymbol{\Phi}^{\cdot 2}\left[\tilde{h}_{1}, \cdots, \tilde{h}_{P}\right]^{T}\right)
\end{aligned}
$$

where ${ }^{2}$ and $\cdot /$ are element by element matrix multiplication and division respectively.

Supposed that the compensation vector $\Delta \mathbf{C}$ contains only one non-zero entry. It means there is only one error in the decoded $\tilde{\mathbf{C}}$ assuming that the solution of MLD is correct. Hence we define $\Delta \mathbf{C}=\left[0, \ldots, \Delta c_{i}, \ldots, 0\right]$ where $i \in[1, \cdots, P]$. The scalar $\Delta c_{i}$ is defined as the $i$ th compensation entry of $\Delta \mathbf{C}$. Hence if $\tilde{\mathbf{C}}$ has one decoding error, (12) can be simplified further as

$$
0<\Delta c_{i}<\boldsymbol{\Lambda}_{i} \quad \text { or } \quad 0>\Delta c_{i}>\boldsymbol{\Lambda}_{i}
$$

where $\boldsymbol{\Lambda}_{i}$ is the $i$ th entry of $\boldsymbol{\Lambda}$. Therefore if we are able to find a $\Delta c_{i}$ satisfying (14), we can use $\Delta c_{i}$ to compensate and correct the $i$ th entry of $\tilde{\mathbf{C}}$ denoted as $\tilde{c}_{i}$. The possible values of $\Delta c_{i}$ are related to the constellation $\mathcal{A}$ and also limited. For example, $\Delta c_{i}= \pm \sqrt{2}$ for QPSK and $\Delta c_{i}=$ $\pm 2 / \sqrt{10}, \pm 4 / \sqrt{10}$, or $\pm 6 / \sqrt{10}$ for 16QAM. Therefore the decoding of $\Delta c_{i}$ becomes another kind of hard decision based on a new constellation. For example, if QPSK is employed, (14) gives us $\Delta c_{i}=\sqrt{2}$ if $\boldsymbol{\Lambda}_{i}>\sqrt{2}$ and $\Delta c_{i}=-\sqrt{2}$ if $\boldsymbol{\Lambda}_{i}<-\sqrt{2}$. Note that the position $i$ is chosen as the position with the largest absolute value of the vector $\boldsymbol{\Lambda}$ so that corrected result is closed with the result from MLD as 


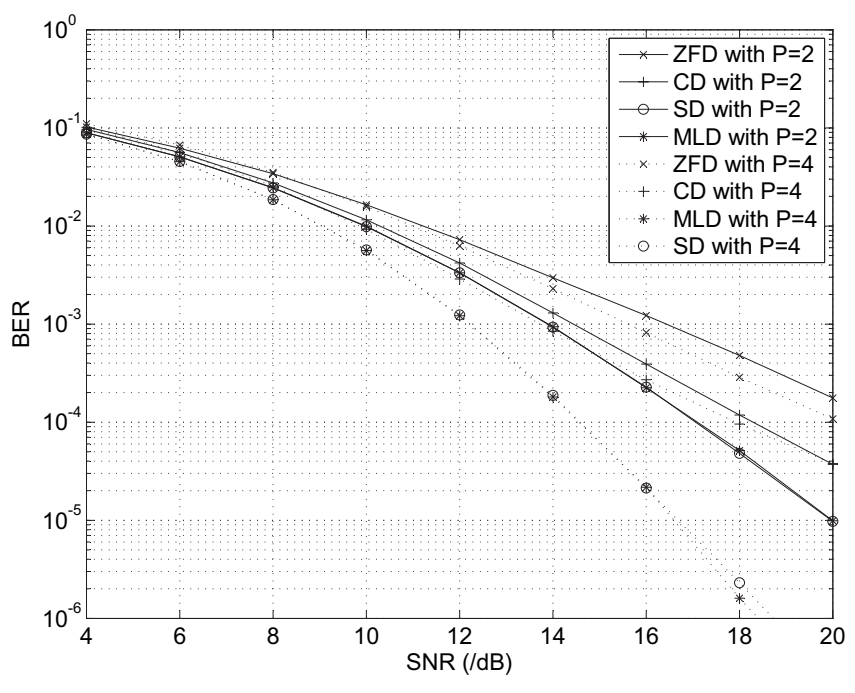

Fig. 1. Performance of MIMO-OFDM system with two or four subcarriers.

much as possible. $\left(\tilde{c}_{i}+\Delta c_{i}\right)$ should be also bounded within the given constellation $\mathcal{A}$, otherwise $\left(\tilde{c}_{i}+\Delta c_{i}\right)$ is out of range.

The advantage of $\mathrm{CD}$ is that the calculation of channel matrix inverse of (3) is simplified as a direct real matrix product in (10), which is similar with ZFD. The real precoding matrices $\boldsymbol{\Phi}, \boldsymbol{\Phi}^{T}$ and $\boldsymbol{\Phi}^{\cdot 2}$ can be stored at the receiver in advance. Only real calculations are involved during the decoding of each subequation in (8). Moreover the decoding of $\tilde{\mathbf{C}}$ and $\Delta \mathbf{C}$ requires two hard decisions in real domain. And the complexity of $\mathrm{CD}$ does not depend on the constellation size of $\mathcal{A}$ except for two hard decisions. The disadvantage of $\mathrm{CD}$ is the lack of full frequency diversity, so that the performance of decoding is worse than MLD and SD. Hence we slightly sacrifice the decoding gain for a faster decoding procedure.

\section{Simulations AND COMPARISONS}

In this section we present simulations to investigate the decoding ability of proposed method. We will compare SD in [9], MLD, ZFD, and the proposed CD. ZFD is the decoding procedure without compensation $\Delta \mathbf{C}$. Hence only (10) is required for ZFD. SD and MLD are based on the equivalent channel equation (3). The constellation of QPSK is employed in every simulation. Fig. 1 is the bit-error-rate (BER) over large number of channel realizations. The random channel is assumed to be a multiray channel. It has uniform power delay profile composed of $L+1$ independent identically distributed complex Gaussian paths with zero mean and equal variance $\left(\frac{1}{L+1}\right)$. We choose $F=128, N_{r}=1$ and $L=3$. The second order characteristics of such MIMO-OFDM is presented in [10]. Considering higher frequency correlation if more subcarriers are employed and practical low and medium SNR scenarios, we focus on the cases of $P=2$ and $P=4$ at $S N R \leq 15 \mathrm{~dB}$.

The performances of ZFD are much worse than CD, MLD and SD in Fig.1. ZFD can not achieve full frequency diversity. However, ZFD can achieve a little improvement in the performance by increasing the number of subcarrriers. Simulations in Fig.1 also show that the proposed CD approaches MLD more than ZFD. It proves that the compensation vector $\Delta \mathbf{C}$ improves the decoding result of ZFD to a certain degree. However full frequency diversity can not be achieved by $\mathrm{CD}$, just as to ZFD. MLD (same as SD) has about $0.5 \mathrm{~dB}$ and $2.5 \mathrm{~dB}$ gains compared with $\mathrm{CD}$ and $\mathrm{ZFD}$ respectively at BER of $10^{-3}$ when $P=2$. Moreover MLD (or SD) has about $1.5 \mathrm{~dB}$ and $3.3 \mathrm{~dB}$ gains compared with $\mathrm{CD}$ and ZFD respectively at BER of $10^{-3}$ when $P=4$. Hence for given BER, the difference between CD and MLD gets larger if more subcarriers are employed. The reason is that most of the decoded $\tilde{\mathbf{C}}$ s with two subcarriers are either error-free or one error. Such situations are suited with the decoding ability of proposed CD. Hence, after taking advantage of characteristics of the precoding scheme, CD presents a tradeoff between system performance and complexity. Therefore it becomes a good candidate for decoding STF codes because of its lowest complexity and relatively good performance for MIMO-OFDM systems at low and medium SNR.

\section{CONCLUSiOnS}

In this paper, we present a novel decoding method for a given space-time-frequency coding scheme. Taking advantage of the simplicity of ZFD, we develop a method to calculate a compensation vector for the output of ZFD. After modification by the compensation vector, the BER performance can be improved significantly. The decoding procedure is relatively simple and independent of the constellation size. Moreover the performance of the proposed decoding method is close to MLD for low to medium SNR range. A trade-off between the system performance and complexity is demonstrated for the implementation of CD.

\section{REFERENCES}

[1] Z. Liu, Y. Xin, and G. B. Giannakis, "Space-time-frequency coded OFDM over frequency selective fading channels," IEEE Trans. Signal Processing, vol. 50, no. 10, pp. 2465-2476, 2002.

[2] Z. Liu, Y. Xin, and G. Giannakis, "Linear constellation precoding for OFDM with maximum multipath diversity and coding gains," IEEE Trans. Commun., vol. 51, no. 3, pp. 416-427, 2003.

[3] W. Su, Z. Safar, and K. R. Liu, "Towards maximum achievable diversity in space, time and frequency: Performance analysis and code design," IEEE Trans. Wireless Commun., vol. 4, no. 4, pp. 1847-1857, 2005.

[4] L. Shao, S. Roy, and S. Sandhu, "Rate-one space frequency block codes with maximum diversity gain for MIMO-OFDM," in Proc. Global Tele. Conf., vol. 2, pp. 809-813, 2003.

[5] A. F. Molisch, M. Z. Win, and J. H. Winters, "Space-time-frequency (STF) coding for MIMO-OFDM systems," IEEE Commun. Letters, vol. 6, no. 9, pp. 370-372, 2002.

[6] C. Tepedelenlioglu, "Maximum multipath diversity with linear equalization in precoded OFDM systems," IEEE Trans. Inf. Theory, vol. 50, no. 1, pp. 232-235, 2004.

[7] Y. Xin, Z. Wang, and G. B. Giannakis, "Space-time diversity systems based on linear constellation precoding," IEEE Trans. Wireless Commun., vol. 2, no. 2, pp. 294-309, 2003.

[8] D. Rainish, "Diversity transform for fading channels," IEEE Trans. Commun., vol. 44, no. 12, pp. 1653-1661, 1996.

[9] K. Su, "Efficient ML detection for communication over MIMO channels," technical report, University of Cambridge, http://www.comm.utoronto.ca/ karen/spheredec.php\#SE, Feb. 2005.

[10] X. Zhu and J. Xue, "On the correlation of subcarriers in grouped linear constellation precoding OFDM systems over frequency selective fading," in Proc. VTC, Spring, vol. 3, pp. 1431-1435, 2006. 